\title{
¿Centralizar o descentralizar los sistemas de información en la empresa?
}

\author{
Amaia Arribas Urrutia \\ Universidad del País Vasco
}

\begin{abstract}
La incorporación de las tecnologías de la comunicación a los sistemas de información en la empresa supone un proceso de aprendizaje organizacional. Las estructuras jerárquicas van desapareciendo gracias a la nueva cultura tecnológica de los directivos, y el departamento de TI asume la responsabilidad de la organización y diseño de los nuevos sistemas de información descentralizados. En definitiva, el papel reestructurador de las TI orienta a las empresas hacia nuevas formas de organización y de gestión documental.
\end{abstract}

\section{Introducción}

as organizaciones se consideran cada vez más como sistemas de infor-
mación. Frente a los recursos tradicionales de la empresa como son el
capital, el material y los recursos humanos, surge con fuerza el recurso
más importante, la información, y su vehículo, los sistemas de informa-
ción.

Los sistemas de información se pueden estudiar desde varios puntos de vista: el de la empresa que necesita el beneficio de los nuevos sistemas de información, el de los profesionales de TI que necesitan comprender mejor la manera de ayudar a la empresa, el de los usuarios que se ven abocados a adoptar una nueva cultura informática, y por último, el del departamento de comunicación interna, que ve en la informática y las telecomunicaciones una herramienta imprescindible para comunicar dentro de la organización.

Abordar en profundidad todas estas áreas en el presente artículo sería imposible. Por ello, trataré los aspectos más importantes de la organización de los sistemas de información de la empresa, sus responsables y el efecto de las nuevas tecnologías de la información en la configuración de los nuevos sistemas de información y en la estructura de la compañía.

Un proyecto de sistemas de información no es un proyecto aislado dentro de la empresa, sino que su diseño engloba a los demás sistemas de la organización, y compromete a los responsables de todos los estamentos de la estructura jerárquica. Es decir, un proyecto de sistemas de información debe de tener en cuenta a 
todo el personal de la empresa, involucrándolo en su diseño. El futuro pasa por una planificación estratégica de los sistemas de información por lo que los directivos deben formar parte de ese proceso sin abdicar en los técnicos.

\section{Organización de los sistemas de información}

La empresa debe valorar cuatro aspectos en el momento de organizar sus sistemas de información:

1. Una compañía que mantenga una estructura organizativa descentralizada debe tener los recursos de información descentralizados también y viceversa.

2. La cultura corporativa en relación a la actitud de los usuarios ante la innovación tecnológica. La alta dirección debe conocer las oportunidades que brindan las tecnologías de la información por lo que necesitan una formación formal e informal sobre ellas mediante comités de gestión cuyos objetivos son los de garantizar la educación necesaria y que todos los miembros la absorban. Ellos serán los profesionales que más van a utilizarla, pero también el personal en general será usuario de las tecnologías por lo que su formación es totalmente necesaria.

3. La evolución en la adopción de la tecnología de la información en la empresa.

4. El impacto estratégico de la nueva tecnología de la información.

Existe una gama muy amplia de posibilidades de centralización y descentralización de los sistemas de información, desde un único ordenador mainframe con pantallas en el mismo local del centro de procesamiento de datos (máxima centralización) hasta un sistema formado por varios ordenadores no conectados (máxima descentralización). Hoy, las empresas optan por una situación mixta o intermedia: un servidor y los demás ordenadores conectados en red a este servidor. Cada ordenador es autónomo pero ofrece la posibilidad de conexión entre los distintos ordenadores de la empresa para compartir datos ${ }^{1}$.

La centralización de los sistemas de información tiene la cualidad de permitir un control más sencillo, ya que es la mejor forma de captar, manipular y usar la información cuando es necesario que un gran número de usuarios puedan acceder a ella. Otra de sus ventajas es que evita la inconsistencia de las aplicaciones y de los programas de los departamentos. Su máximo inconveniente radica en que genera retrasos y pérdidas de tiempo al establecer prioridades entre usuarios, a la vez que anula la iniciativa individual. 1 El sistema de información de una organización no es sólo un centro de proceso de datos sino que éste forma parte de los recursos de información; sería una parte de las actividades de información y una parte de las relaciones, de las estructuras y fines que tiene que ver con el soporte físico del sistema de información. También Le Moigen (1973) participa de esta idea señalando que el sistema de información no es sólo un sistema de contabilidad. 
En cambio, la descentralización permite adaptar las necesidades del usuario, por lo tanto, motiva al usuario, facilita el reparto de las tareas y multiplica la eficacia de las funciones directivas. El centro de información pasa a tener una función consultora ${ }^{2}$. El gran inconveniente es que la tecnología no satisface siempre en coste y calidad a los requerimientos de cada usuario.

En un principio, la descentralización era obligada ya que los ordenadores apenas podían manipular la carga de trabajo de un único departamento. Con el desarrollo de los mainframes y de las redes terminales provocaron la centralización de las aplicaciones y de las bases de datos. Después se volvió a la descentralización con los miniordenadores, ya que con la tecnología de los sistemas abiertos la informática departamental, de grupo y de usuario final se hizo una realidad. Como consecuencia de ello, se trasladó las bases de datos y especialistas de la información a algunos departamentos, así como la creación de centros de información para apoyar a la informática de usuario final. La última tendencia es la de controlar más los recursos de información de una empresa. El resultado ha sido volver a la centralización, y en otras ocasiones, un desarrollo de estructuras híbridas con componentes centralizados y descentralizados.

El hardware ofrece alternativas para la descentralización en diversas áreas:

1. Capacidad de procesamiento. Con la instalación de varios procesadores es posible que cada usuario tenga el suyo. El ordenador central queda liberado, sobre todo en ocasiones donde el usuario demanda por mucho tiempo el ordenador, y también en las ocasiones que requiere liberar líneas de comunicación al tratarse de ordenadores situados en distintos locales.

2. Con la descentralización de los programas y datos, el funcionamiento de los usuarios gana en independencia, dependiendo menos del equipo central. Pero surge el problema de redundancia cuando los datos deben ser compartidos por varios usuarios. Por tanto, esta descentralización sólo es recomendable cuando sean datos que no vayan a ser compartidos o cuando el alto volumen de la base de datos y su distribución geográfica lo requiera.

3. En cuanto a los usuarios, este problema está casi resuelto ya que hoy día cada usuario trabaja en su propio ordenador.

4. La descentralización física planteada de modo distinto según el escenario. Por un lado en locales separados por vías públicas, y por otro, dentro de un mismo local donde prevalecen los factores como el tipo de perfil del trabajo de los usuarios (demanda de tiempo de procesador, rapidez de tiempo de respuesta, necesidad de compartir información con otros usuarios) o aspectos económicos.

2 Los centros de información deben de informar a los usuarios de los servicios que les ayudarán a ayudarse Estos centros son unos mecanismos organizativos para la implantación de la informática de usuarios final. Cuanto más aumente la descentralización y el downsizing, estos centros irán perdiendo protagonismo, sustituyéndoles los especialistas en apoyo a los usuarios finales, departamentos y grupos de trabajo. 
Según García Bravo, el equilibrio entre el equipo multiusuario y la red local, conforma las características de cada empresa. Lo ideal sería mantener una centralización en el terreno técnico y una descentralización en los usuarios. Las ventajas de la descentralización de usuarios estriban en que éstos interactúan con el ordenador satisfaciendo sus necesidades de manera más eficaz, mientras decrecen los costes de hardware y software. Tampoco hay que olvidar la existencia de varios procesadores independientes, así como la independencia de análisis y diseño de cada unidad, y por último, la gestión de las bases de datos y aplicaciones en cada departamento. Como desventaja hay que señalar la posible incompatibilidad entre hardware, software y sistemas, y que los precios de los costes de comunicación se disparen.

$\mathrm{Al}$ dilema de la centralización o descentralización de los sistemas de información, Monforte propone la llamada informática distribuida. Esta tecnología es posible gracias a las posibilidades de interconexión de las telecomunicaciones. Mantiene un equilibrio entre la centralización y la descentralización. Se desarrollan aplicaciones específicas para determinados puestos de trabajo que mediante su interconexión, facilita el intercambio de información siempre que se necesite. Se trata de una arquitectura mixta donde sólo se descentraliza la dependencia funcional, mientras que las demás unidades siguen unidas al departamento central. Pero hoy ya no sólo es descentralización funcional sino geográfica también, aun manteniendo la dependencia de las personas y el control centralizado en el departamento de informática.

La tendencia actual es, lo que llama García Bravo "descentralización comunicada", es decir, la proliferación de miniordenadores y microordenadores departamentales o de grupo.

\section{Organización descentralizada}

El impacto de los sistemas de información en el diseño organizativo es indudable. Desempeñan un papel fundamental en el proceso de difundir la información necesaria para las diferentes unidades donde se toman las decisiones. Contribuye además a proporcionar la flexibilidad necesaria para las fuentes de información y los programas de recopilación de la información.

Existe la necesidad, por tanto, de un proceso de reorganización para una buena administración del recurso información, una reorganización donde prime la optimización de los flujos de información por sus sistemas por encima de las jerarquías, donde haya más modelos orgánicos y menos mecánicos (Claver Cortés y García Bravo, 1997: 76).

La nueva tecnología contribuye al allanamiento de la pirámide jerárquica ${ }^{3}$,

3 El crecimiento de los sistemas de información dentro de la empresa provoca cambios en los criterios organizativos. El estrechamiento de la pirámide de decisión hace prever una complejidad de los sistemas de información dado el desplazamiento hacia arriba de las aplicaciones que haya que desarrollar. Cada vez existe un mayor peso del nivel táctico, impulsado por la descentralización en la ejecución de las operaciones. 
y absorbe algunas de las tareas de los mandos medios. Resulta una pirámide no sólo achatada sino extendida (Luna, Martínez y Salmerón, 1999: 172-173) ya que se dispone de elementos técnicos para trabajar no sólo fuera de la oficina sino en cualquier parte del mundo ${ }^{4}$. Ello implica que conceptos en la gestión de los sistemas de información sean revisados y se tengan que materializar en cualquier organización sea del tamaño que sea.

La nueva estructura empresarial está formada en su mayoría por especialistas o trabajadores del conocimiento provistos de estaciones de trabajo informáticas y provistos de toda la información que precisen. Como ya decía Drucker (1988), en la empresa basada en la información, los conocimientos estarán en las mentes de los especialistas que realizarán sus trabajos en la parte baja de la pirámide jerárquica. Por tanto, no tiene ningún sentido negarles la información y capacidad de autodirección, y se puede afirmar las estrechas relaciones entre las nuevas tecnologías de la información y las estructuras organizativas. ${ }^{5}$

Cada miembro de la empresa debe de tener claras sus responsabilidades en la organización. Así, la alta dirección debe involucrarse directamente en los problemas de los sistemas de información y comprender que no son exclusivos del departemento de informática, así como incentivar las iniciativas relacionadas con las tecnologías de la información. Los usuarios deben tener un espíritu participativo, colaborar con los equipos de desarrollo informático y cumplir las normas, la seguridad y la aplicación de la documentación. Por último, los informáticos no deben caer en el uso de tecnicismos, fijar las normas de uso de los sistemas de información y ser constante en la formación de los usuarios.

Las nuevas tecnologías de la información modifican las organizaciones ya que se observa una disminución de mandos intermedios por la reducción de niveles jerárquicos y aplanamiento de los organigramas. La pirámide de gestión de Anthony, donde el subsistema operativo está en la base, el táctico en el centro y la alta dirección en la cúspide, está en crisis. Cambios externos provocan cambios internos en la gestión y organización empresarial ${ }^{6}$.

Los sistemas de información y comunicación reemplazan a la jerarquía con una redefinición de los puestos de trabajo y se eliminan o suavizan las tareas rutinarias. Se obtiene información determinada mediante las TI sin necesidad de

4 El avance tecnológico ha traido además el teletrabajo y el outsourcing que hasta ahora había estado reservado a determinado personal debido a la independencia que supone este tipo de trabajo, y ahora se ha extendido a casi todos los niveles empresariales.

5 Huber y McDaniel (1986) contribuyen a este pensamiento añadiendo el consejo de emplear las nuevas tecnologías de la información para aumentar la disponibilidad de la información en las unidades decisoras, a la vez que decrece el tamaño y el número de éstas.

6 Un estudio realizado por el profesor Francisco Martínez López en 1992 analizó el consumo de información Un estudio realizado por el profesor Francisco Martínez López en 1992 a sizó el consuno de información en la empresa y concluyo que la informática transaccional (la de los subsistemas operativos) va perdiendo información en el nivel táctico y en menor medida el estratégico, era mayor 
intermediarios. Se refuerza las posibilidades de coordinación directa por intercambio mutuo de información sin mandos jerárquicos intermedios, con lo que la comunicación entre los departamentos es mucho más fluida, incluso se pueden llegar a fusionar si la relación es muy estrecha. La facilidad para intercambiar información no quiere decir que todos los usuarios tengan libre acceso a todo tipo de información. Se trata de facilitar la información necesaria para el trabajo de cada uno, y limitar el uso de informes en papel ya que tardarían más tiempo en ser insertados en documentos electrónicos.

En cuanto al usuario, será más cualificado al tener que utilizar como herramienta de trabajo las TI, y le hace más participativo ya que se desinhibe al usar un medio más impersonal. Se descentralizan las decisiones ya que la información es más asequible a los usuarios en general. Las TI se vuelven un elemento democratizador ya que facilita el sentido ascendente de la información a través de la jerarquía de la empresa.

Son aquellas empresas que poseen un departamento o responsable de tecnologías de la información las que acusan estas tendencias, ya que las organizaciones que no disponen de una unidad responsable de informática apenas presentan estas características, sin olvidar que que casi ningún responsable desea delegar decisiones ni descentralizar.

\section{El Departamento de TI}

La creciente importancia de la información, junto con la mayor distribución de la tecnología a los usuarios informáticos, hace que los profesionales de las tecnologías de la información y los propios directivos de la empresa consideren el nuevo papel de los departamentos de $\mathrm{TI}^{7}$ de la compañía.

Se producen dos situaciones en muchas empresas actualmente. Por un lado, en el organigrama funcional de la empresa, el departamento de sistemas de información (todavía llamado de informática o de proceso de datos) se sitúa en una posición dependiente de los servicios administrativos. Por otro lado, se crea una barrera de comunicación entre los estamentos directivos de la empresa y la jefatura del departamento de sistema de información. Y por último, la conexión entre los objetivos y los planes del sistema de información es inexistente, y cuando existe se lleva a cabo a través de las peticiones de los usuarios.

7 Un estudio realizado por la revista WeWeek revela que la mayor parte de las 500 empresas de Fortune ya disponen de departamentos especializados en la Red. Son relativamente pequeños, y la mitad de las empresas encuestadas tienen entre dos y cinco personas asignadas a esos departamentos, aunque también cerca del $12 \%$ de esos departamentos tienen veinte o más personas.

En este estudo aprón doces En este estudio aparecía docenas de especialidades que no era fácil de encontrar hace unos poco años antes. Estas son la de especialstas en de Internet, administrador de seguridad, director de nuevos medios, director de servicios de Internet, técnico multimedia y desarrollo de contenidos en línea. También hay otros puestos más creativos como
cibercomandante, maestre de la Red mago dela Red y diosa de la Red. 
Tradicionalmente han sido los técnicos del Departamento de Informática quienes se han encargado de desarrollar los sistemas de información, de acuerdo con los responsables de las operaciones que les indicaban sus necesidades. La planificación de estos sistemas se ha concebido fuera del plan estratégico de la empresa. La alta dirección ha intervenido muy poco en su definición y desarrollo, y su participación se ha reducido a aprobar o rechazar las inversiones propuestas por los técnicos. Es decir, como los directivos carecían de formación técnica, se han visto en la obligación de delegar en los informáticos. Pero, la tecnología de la información es demasiado importante para dejársela a los informáticos, es decir, la TI se está convirtiendo en algo tan importante que no se puede delegar en los informáticos sino que los directivos de la empresa deben encargarse de su gestión (Fernández, 1994: 98).

El papel de los directivos de tecnologías de la información es el de asegurar que los directivos conozcan el potencial y los recursos de utilización de la TI, asî como proporcionar asesoría y conocimientos técnicos para el funcionamiento de las estrategias empresariales. Más que la organización de la TI lo que más importa es la capacidad de los directivos para utilizarlas correctamente.

La posición del departamento de sistemas de información en el organigrama debe estar de acuerdo con el diseño de las unidades estratégicas de tecnologías y sistemas de información, y dotado de mecanismos organizativos para que cada responsable de cada unidad mantenga el control sobre sus recursos de tecnologías de la información. Pero es fundamental que el departamento de tecnologías y sistemas de información no dependa de la dirección correspondiente al usuario principal para evitar así los sesgos que pudieran existir en la distribución de los recursos al resto de la empresa.

En relación a la centralización de técnicos, la administración de datos está bajo control de un sólo departamento especializado, bajo la dirección de un gerente, normalmente de línea. Pero su gran inconveniente es alejar a los usuarios de los sistemas de información.

La evolución hacia la descentralización, ¿podría causar la desaparición del departamento de informática?. Un paso hacia la descentralización es que el departamento de informática establezca las políticas de adquisición y desarrollo de nuevas tecnologías y asegure el intercambio de información. No hay que olvidar tampoco que, a mayor preparación del usuario medio, mayor presión hacia la descentralización. Lo cierto es que aún queda muy lejos el día en que los usuarios tengan el control de sus sistemas individuales de información. Una descentraliza-

8 La organización de la tecnología de la información en la empresa exige la coordinación de la estrategia de TI con la estrategia empresarial de la organización. Cuando se debaten estrategias empresariales, los responsables del departamento de TI deben estar presentes, contribuyendo éstos a los planes de gestión. 
ción absoluta supondría que el usuario es un especialista en sistemas de información, lo que hoy parece improbable 9 .

\section{El responsable del sistema de información. El CIO.}

El responsable de sistemas de información en la empresa juega un papel fundamental ya que su misión consiste en crear un contexto adecuado para que se dé la colaboración entre el personal de sistemas de información y personal de negocio. Hay una serie de recomendaciones a seguir para crear este contexto:

1. Explicar los costes de los sistemas de información.

2. Buscar la colaboración con las distintas unidades de negocio. Mantener esa colaboración exige por un lado, estar dispuesto a aprender mutuamente, es decir, compartir conocimiento, definir las responsabilidades, respetar las competencias de cada miembro, e institucionalizar esas relaciones de colaboración creando vínculos organizativos.

Desde la más altas instancias también se puede animar y potenciar esta colaboración dotando de formación tanto en tecnología a los empleados, la creación de equipos de trabajo, como diseñando políticas de recursos humanos que potencien esta colaboración.

El objetivo de los profesionales de la informática o del proceso de datos, que es el garantizar el funcionamiento de los medios informáticos, se ve dificultado por el hecho de que todavía algunos directivos se encuentran, por falta de aprendizaje, con el problema de tener que decidir con la información que esta tecnología le proporciona. Además, el diálogo entre quienes llevan el proyecto informático y la alta dirección no es muy fluido, unos recelos que van desapareciendo con la nueva cultura empresarial.

3. Mostrar la capacidad sobre la tecnología aplicada, ganándose así la credibilidad. Las tecnologías de la información son herramientas caras con valor estratégico para la empresa, y que si son gestionadas adecuadamente ofrecen una ventaja competitiva.

En cuanto a su estructura interna las funciones que debe acometer son las siguientes:

1. Seguridad de los sistemas e instalaciones.

2. Administración de la información. Se trata de gestionar los ficheros de las bases de datos y el mantenimiento de un catálogo de datos y de una biblioteca documental.

3. Planificación de recursos. El departamento de informática define las líneas que hay que seguir en la aplicación, incoporación e integración de nuevas tecnologías en el proceso de información.

9 Creo que es impensable la desaparición del departamento de informática ya que siempre será necesario establecer políticas generales de los sistemas de información. 
La descentralización en la toma de decisiones implica la distribución de la capacidad informática y la disolución de los departamentos de informática, que eran auténticas fortalezas de poder en algunas empresas, y va surgiendo un nuevo concepto: el centro de informacón corporativo (Monforte, 1994: 17). Ahora en los organigramas empresariales aparece una figura nueva, el responsable de los sistemas de información, el $\mathrm{CIO}^{10}$ (Chief Information Officer), dándole un carácter de gestor más que de ténico. Su función es la de integrar las decisiones y planes de sistemas de información en la estrategia empresarial.

El CIO es el encargado de la administración de los sistemas de información. Su papel se acerca más al de management que al de técnica informática. Las cualidades más importantes que debe poseer un CIO son (Monforte, 1995: 189190) la de ser una persona con carisma dentro de la empresa; un estratega capaz de planificar los recursos de información; un innovador preocupado por la evolución de las tecnologías de la información; un gestor de la información, responsable de la integración de los recursos informáticos y la estrategia de la empresa; e interlocutor entre la alta dirección y los usuarios.

Synott (1981) habla del CIO, como un alto ejecutivo cuya responsabilidad principal era la gestión de la información., y cuyas atribuciones iban más allá de las que hasta entonces había tenido el jefe de informática. Así como el jefe de informática había surgido como consecuencia de la incorporación de ordenadores a las empresas, el CIO debía su existencia a la descentralización de los departamentos de informática. Sus funciones principales eran la coordinación de recursos informáticos distribuidos, la definición y mantenimiento de la arquitectura informática de la empresa y la asignación de recursos económicos entre las distintas unidades bajo su responsabilidad. Además, tenía una misión estratégica: conseguir que las tecnologías contribuyeran al aumento de la productividad y sirvieran para identificar nuevas ventajas competitivas. El CIO debe conseguir la alineación de la estrategia informática con la estrategia de la empresa, y no sólo velar por el funcionamiento de las aplicaciones informáticas.

La consolidación de la figura del CIO en la empresa supone el reconocimiento de la importancia de administrar la información como recurso corporativo, y de que en su área de decisión descansa el futuro de la empresa.

Este nuevo cometido, más supradepartamental, le libera en principio de la dependencia directa de otros departamentos (financiero... ). El CIO tiene que ser más independiente y disponer de un nexo más directo con los estamentos responsables de de la planificación estratégica. Para ello, nada mejor que disponer de información de primera mano sobre los objetivos y estrategias de la empresa.

10 Synott predijo que las oportunidades laborales para los CIO’s eran extraordinarias debido a la multiplicación de sistemas informáticos descentralizados. Se estimó que cerca del cuarenta por ciento de las mi mayores empresas de ranking elaborado en 1989 por el semanario Business Week (26 de febrero de 1990, pag 78-80),disponían de un CIO 
6. Nuevas tecnologías para la descentralización de los sistemas. Intranet.

Durante treinta años el mercado ha hablado de la necesidad de que las organizaciones descentralicen la decisión de hacer y reestructurar el modelo tradicional de toma de decisión centralizado. Es ahora cuando la tecnología empieza a tener importancia a nivel de reducción de papel y de involucración de usuarios en actividades de trabajo en grupo.

Finalmente ha llegado la tecnología que permite a las empresas apoyarse en una toma de decisiones descentralizada y que coordina actividades. Su adaptación es fácil y barata para esas empresas que inicialmente investigaron sobre la arquitectura cliente-servidor, ya que ellas tenían la infraestructura técnica instalada. Web solamente proporciona el software para imponer una tendencia que ya había empezado a funcionar.

Ahora, el concepto de intranet conlleva un número cada vez mayor de normas y herramientas que soportan las comunicaciones abiertas de red. Muchas de las herramientas que actualmente existen en el mercado se desarrollaron originalmente en entornos propietarios, modificándose para saltar al vagón Web. Idealmente se ha adaptado el Web para soportar una infraestructura de coordinación y comunicación en un entorno distribuido de toma de decisiones.

Internamente, muchas de las grandes empresas tienen los mismos problemas de información heterogénea y distribuida dentro de sus organizaciones que la tecnología Internet y Web podrían resolver bajo ciertas condiciones. Y es fácil para muchas de estas organizaciones, identificar los verdaderos beneficios al reemplazar los procesos escritos por el flujo de información electrónica. Ya que las redes son internas, las normas de seguridad y de acceso que se pueden imponer sería imposible sobre una red pública.

\section{Nueva cultura tecnológica y organizativa}

El papel del centro de información corporativo (Monforte, 1995: 182), el antiguo departamento de informática, cobra un gran protagonismo ya que soporta la supervisión de la calidad de las aplicaciones y el mantenimiento de las posibilidades de intercambio y compatibilidad.

La situación del centro de información en el organigrama de la empresa es cada vez más cercana a la alta dirección, es un staff a presidencia, debido a la creciente necesidad de contar con información de gestión de gran calidad y al progresivo carácter estratégico de las tecnologías de la información.

En definitiva, las organizaciones de hoy tienen que ser más orgánicas, y se va imponiendo la flexibilidad a través de las formas reticulares que, poco a poco, se van adaptando inducidas por las nuevas tecnologías.

Uno de los grandes problemas con los que ha de enfrentarse el gestor es la cultura centralizadora que existe casi siempre en el Centro de Proceso de Datos (CPD). Desde el comienzo y debido a razones tecnológicas, las actividades del 
CPD han sido claramente centralizadoras, debido en parte por la mitificación que ha existido en torno al ordenador, constituyendo esta mitificación una fuente de poder (Martín, 1995: 153).

La tarea básica de la dirección de la nueva organización es la de descentralizar los procesos de toma de decisiones y suavizar las estructuras jerárquicas, dando mayores responsabilidades a los trabajadores. Esto implica que los empleados necesitan mayor información e interactuar con sus superiores.

En algunas organizaciones la cultura empresarial penaliza a aquellos empleados que no participan en equipo, es decir, que no comparten información. Por tanto, las personas están deseosas de compartir conocimientos y cooperar ya que su retribución está ligada a este mosaico de relaciones con sus compañeros de empresa.

Hasta ahora, en la cultura de empresa prevalecía la estructura y comportamiento rígido, pero el paso hacia sistemas económicos abiertos favorece la necesidad de abrir y agilizar sus diseños organizativos. Asistimos por tanto, al fin de las estructuras jerarquizadas y estáticas, caracterizadas por la fragmentación y segmentación de la empresa en unidades funcionales alejadas entre ellas. Ahora, aparecen nuevas formas organizativas caracterizadas por su flexibilidad, rapidez y descentralización.

Con la descentralización de los sistemas de información de una empresa, los intercambios de información fuera de la jerarquía son más frecuentes, lo que no significa que las decisiones no sean tomadas por los dirigentes, sino que la organización debe dotarse de un sistema de información tan independiente como le sea posible para hacer frente a las restricciones organizativas, y que sea válido sin que tenga que modificarse continuamente.

De todas maneras, ninguna organización es la panacea ya que en muchas empresas coexisten formas muy diferentes de organización. La cuestión es aplicar la correcta en el sitio y momento más oportuno.

\section{Conclusión}

Los sistemas de información son el espejo de la organización. El triángulo formado por la información, el conocimiento y las nuevas tecnologías está afectando a la forma de organizar los sistemas de información y a la estructura organizativa de la empresa.

Centralizar o no los sistemas de información es una solución a medida de cada empresa. Es decir, cualquier actuación debe tener en cuenta la naturaleza de la empresa, su tamaño, su tecnología y complejidad. Lo ideal es adoptar lo mejor de cada solución y evitar aquellas acciones que nos conduzcan a caminos sin retorno y a la vuelta a una organización jerárquica donde la información fluye en un único sentido (de arriba a abajo), y donde el usuario pierde su papel activo. 
Sucede en numerosas ocasiones, que las estructuras de las empresas tiemblan con el sólo el hecho de pensar en cambiar los sistemas de información. Prefieren cambiar el resto: su organización, funciones, sistema de retribución incluso la cultura de la empresa antes que abordar una transformación de tal magnitud. Sobre esta situación Harold Leavitt propone el equilibrio entre la estrategia de una empresa, su estructura, su personal y sus funciones y su tecnología. Si alguna de estas variables cambia, deben modificarse el resto para mantener el equilibrio. Los sistemas de información tienen mucho que decir en este escenario hasta tal punto que no se sabrá si fue antes los sistemas de información o las nuevas tendencias organizativas. Los directivos deben ser valientes y apostar por el cambio de sus sistemas de información, apoyados por la evolución de las nuevas tecnologías de la información, para definir la empresa del siglo XXI. El éxito o el fracaso del empleo de las TI en los sistemas de información en una compañía depende en gran medida de la capacidad que tengan los directivos para entender las posibilidades de los recursos de las TI.

Sistemas de información, nueva cultura tecnológica y cambios organizativos caminarán de la mano durante los próximos años.

\section{Bibliografía:}

- ALIN, Fréderic; LAFONT, Denis y MACARY, Jean-Francois: El proyecto intranet, trad. Amadeus Brugués, edit.Gestión 2000, Barcelona, 1997.

- ANDREU, Rafael; RICART, Joan y VALOR, Josep: Estrategia y sistemas de información, Serie Mc Graw-Hill de Management, Madrid, 1998.

- ARTECHE, Miguel: "El 60\% de las empresas consideran claves los sistemas de información", en EXPANSION, 4 de junio. Madrid, 1999.

- CLAVER CORTÉS, Enrique y GARCÍA BRAVO, Daniel: "Reflexiones en torno a la dimensión estratégica de las tecnologías de la información en la empresa”, en Esic-Market, núm. 95, enero-marzo, Madrid, 1997.

- CLAVER CORTÉS, Enrique y GONZÁLEZ RAMÍREZ, María de los Reyes: "Los sistemas y tecnologías de la información: su repercursión en las estructuras empresariales", en Alta Dirección, núm. 198, mayo-junio, Barcelona, 1998.

- DEL VAL PARDO, Isabel: "Modernidad y postmodernidad del diseño organizativo", en Esic-Market, núm. 100, mayo-agosto, Madrid, 1998.

- DRUCKER, Peter: "The coming of the new organization", en Harvard Business Review, jan-febr, 1988.

- EATON, J. y BADWEN, D: "What kind of resource is information?", en International Journal of Information Management, núm. 11, 1991.

- EDWARDS, Chris; WARD, John y BYTHEWAY, Andy: Fundamentos de sistemas de información, ed. Prentice Hall, Madrid, 1998. 
- FERNÁNDEZ, José Antonio: "La tecnología de la información, factor estratégico en la segunda mitad de los 90", en Harvard Deusto Business Review, núm 64, noviembre-diciembre, ed. Deusto, Bilbao, 1994.

- GAIRIN SALLÁN, Joaquín: "La dirección en los procesos de aprendizaje colectivo", en Alta Dirección, núm.191, enero-febrero, Barcelona, 1997.

- GARCÍA BRAVO, Daniel: Sistemas y tecnologías de la información en la empresa, ed. Instituto de Cultura Juan Albert, Alicante, 1995.

- GONZÁLEZ DE RIVERA, Luis: “Aprender a trabajar en equipo: clave de las organizaciones que aprenden”, en Alta Dirección, núm. 191, enero-febrero, Barcelona, 1997.

- LUNA, Paula; MARTÍNEZ Francisco José y SALMERÓN, José Luis: "Los sistemas de información y la nueva arquitectura empresarial”, en Esic-Market, núm. 103, mayo-agosto, Madrid, 1999.

- MONFORTE, Manfredo: Sistemas de información para la dirección, Ed. Pirámide, Madrid, 1995.

- ORTEGA MARTÍNEZ, José Antonio: "Ventaja competitiva y sistemas de información: un enfoque estratégico", en Harvard Deusto Business Review, núm. 81, noviembre-diciembre, ed. Deusto, Bilbao, 1997.

- QUINTÍN MARTÍN, Martín: "Sistemas de información. Su aplicación a la empresa.”, en Esic Market, núm. 70, octubre-diciembre, Madrid, 1990.

QUINTÍN MARTÍN, Martín: La informática como elemento dinamizador de las nuevas tecnologías en la empresa", en Esic Market, núm. 88, abril-junio, Madrid, 1995.

- QUINN, James; ANDERSON, Philip y FINKELSTEIN, Sydney: "La gestión del intelecto profesional: sacar el máximo de los mejores", en Harvard Deusto Business Review, núm. 75, noviembre-diciembre. Edic. Deusto, Bilbao, 1996.

ROCKART, John; EARL, Michael y ROSS, Jeanne: "Ocho imperativos para la nueva organización de la tecnología de la información", en Harvard Deusto Business Review, núm. 80, septiembre-octubre, ed. Deusto, Bilbao, 1997.

- SCHWARTZ, Evan: "El efecto de la red: cómo está cambiando la vida que conocemos", en Harvard Deusto Business Review, núm. 81, noviembre-diciembre, ed. Deusto, Bilbao, 1997.

- SYNNOT, W., y GRUBER, W.H.: Information Resource Management-Opportunities and strategics for the 1980's, de. John Wiley, Nueva York, 1981. 